\title{
Evoluzione dell'ipotiroidismo subclinico e sua correlazione con le concentrazioni sieriche di glucosio e trigliceridi in soggetti affetti da obesità morbigena dopo intervento di chirurgia bariatrica di gastrectomia a manica
}

\author{
Paola Fierabracci
}

Pubblicato online: 2 aprile 2014

(C) Springer International Publishing AG 2014

\section{Commento a:}

Evolution of subclinical hypothyroidism and its relation with glucose and triglycerides levels in morbidly obese patients after undergoing sleeve gastrectomy as bariatric procedure.

J. Ruiz-Tovar, E. Boix, I. Galindo, L. Zubiaga, M. Diez, A. Arroyo, R. Calpena.

Obes Surg (2013) doi:10.1007/s11695-013-1150-5

[Epub ahead of print]

Lo scopo dello studio era di valutare l'evoluzione dell'ipotiroidismo subclinico $(\mathrm{SCH})$ in soggetti obesi dopo l'intervento di gastrectomia a manica (sleeve gastrectomy, SG) e la sua associazione con il calo ponderale e i parametri biochimici di rischio cardiovascolare. È stato condotto uno studio retrospettivo su 66 pazienti con obesità morbigena che erano stati sottoposti a SG tra l'ottobre 2007 e il novembre 2012 presso l'Unità di Chirurgia Bariatrica dell'Ospedale Universitario di Elche (Alicante, Spagna). Venivano valutati il calo ponderale, l'indice di massa corporea (IMC) e l'eccesso ponderale, la glicemia basale, il profilo lipidico e le concentrazioni sieriche del TSH prima e a distanza di 3, 6 e 12 mesi dall'intervento. La prevalenza di SCH era del 16,7\% prima dell'intervento, del $10 \%$ a 3 mesi, del $3,3 \%$ a 6 mesi e scendeva all' $1,7 \%$ a 12 mesi. Veniva riscontrata una correlazione significativa tra il decremento del TSH e il calo ponderale a 12 mesi, e la riduzione del TSH si correlava con la riduzione della glicemia e dell'emoglobina glicata a partire dal sesto mese dopo l'intervento chirurgico. Riguardo

P. Fierabracci $(\varangle)$

UO Endocrinologia I, Azienda Ospedaliero Universitaria Pisana, Pisa, Italia

e-mail: pfierab2001@yahoo.it al profilo lipidico non veniva riscontrata alcuna correlazione tra il decremento del TSH e la riduzione del colesterolo totale, LDL o HDL, mentre veniva rilevata una correlazione significativa tra la riduzione del TSH e il decremento dei trigliceridi e dell'indice di rischio cardiovascolare (rapporto trigliceridi/colesterolo HDL). Gli autori concludono che l'ipotiroidismo subclinico dei soggetti affetti da obesità grave migliora dopo chirurgia bariatrica mentre non vi sono correlazioni significative nelle variazioni delle concentrazioni sieriche di colesterolo totale e LDL, suggerendo che nei soggetti con obesità grave l'ipotiroidismo subclinico è una conseguenza dell'eccessivo accumulo di tessuto adiposo e non una condizione di ipotiroidismo reale.

È noto dalla letteratura che nell'obesità grave vi è un incremento delle concentrazioni sieriche del TSH, ma questa alterazione biochimica è un fenomeno secondario all'obesità piuttosto che una condizione di reale ipotiroidismo. È stato ipotizzato che l'ipertireotropinemia conseguente all'eccessivo accumulo di tessuto adiposo sia un meccanismo termogenetico adattativo o, in alternativa, che il meccanismo di feedback sulla secrezione di TSH da parte degli ormoni tiroidei possa essere alterato nell'obesità. Lo studio di Tovar e collaboratori confermerebbe questa ipotesi, in quanto dopo il calo ponderale si verifica un decremento delle concentrazioni sieriche del TSH e ciò è indipendente dal miglioramento dei vari parametri biochimici della sindrome metabolica. Sulla base della nostra esperienza riteniamo, comunque, fondamentale che in tutti i pazienti da sottoporre a chirurgia bariatrica venga effettuato uno screening dell'autoimmunità tiroidea al fine di selezionare una condizione di ipotiroidismo lieve di origine autoimmune, che necessita di una terapia ormonale sostitutiva prima di procedere all'intervento. 